hep-th/9908060

DAMTP-1999-105

MRI-PHY/P990824

\title{
Non-supersymmetric D-Brane Configurations with Bose-Fermi Degenerate Open String Spectrum
}

\author{
Matthias R. Gaberdiel to \\ Department of Applied Mathematics and Theoretical Physics \\ University of Cambridge, Silver Street \\ Cambridge, CB3 9EW, U.K. \\ and
}

Ashoke Sen

Mehta Research Institute of Mathematics

and Mathematical Physics

Chhatnag Road, Jhoosi, Allahabad 211019, INDIA

\begin{abstract}
The spectrum of open strings on various non-BPS D-brane configurations in type II string theory on a K3 orbifold is analysed. At a generic point in the corresponding moduli space the spectrum of open strings does not have any degeneracy between bosonic and fermionic states. However, there exist special values for these moduli for which many nonBPS D-brane configurations have an exactly bose-fermi degenerate open string spectrum at all mass levels. In this case the closed string exchange interaction between a pair of such D-brane configurations vanishes at all distances.
\end{abstract}

*E-mail: M.R.Gaberdiel@damtp.cam.ac.uk

${ }^{\dagger}$ E-mail: asen@thwgs.cern.ch, sen@mri.ernet.in 


\section{Contents}

\begin{tabular}{llr}
\hline & Introduction and Summary & 2
\end{tabular}

2 Examples of non-BPS D-brane configurations with exact bose-fermi de$\begin{array}{ll}\text { generacy } & 6\end{array}$

$2.1 \quad$ Example 1: BPS D-brane wrapped on non-supersymmetric cycle of K3 . . 7

2.2 Example 2: Pair of BPS D-branes wrapped on supersymmetric cycles of K3 11

\begin{tabular}{|llll}
\hline 3 & The boundary state approach & 13
\end{tabular}

3.1 Example 1: BPS D-brane wrapped on non-supersymmetric cycle of K3 . . 14

3.2 Example 2: Pair of BPS D-branes wrapped on supersymmetric cycles of K3 17

3.3 Example 3: A pair of BPS D-branes wrapped on non-supersymmetric cycles

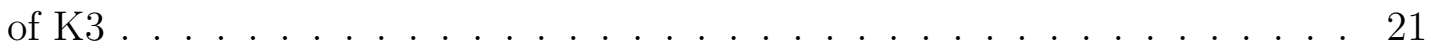

3.4 Example 4: A pair of BPS D-branes wrapped on supersymmetric cycles of

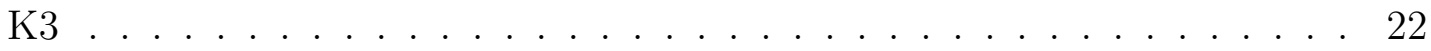

\section{Introduction and Summary}

BPS D-branes carrying identical charges do not exert any force on each other, and can be at equilibrium at all distances. This is a consequence of supersymmetry, and reflects the fact that the spectrum of open strings living on the world volume of the system has exact degeneracy between bosonic and fermionic states at all mass levels. As a result the partition function of the open strings, which corresponds to the negative of the interaction energy of the pair of D-branes, vanishes identically.

If we consider a set of non-BPS D-branes, or a system of BPS D-branes carrying different sets of charges and/or with different orientations so that the combined system is not supersymmetric, then in general the spectrum of open strings will not have exact bose-fermi degeneracy. The open string partition function, and hence the interaction energy of the D-branes, is then not zero. In this case the D-branes exert a force on each other, and the system is not in equilibrium.

In this paper we analyse explicitly the partition function of open strings for various non-supersymmetric systems of D-branes in type IIA/IIB string theory on an orbifold K3. We find that in general this partition function does not vanish and that it has non-trivial dependence on the relative distance between the branes. However, we also find that as we vary the moduli of the K3 orbifold, the open string spectrum can develop exact bosefermi degeneracy at some special points in the moduli space. For such configurations the 
partition function vanishes identically, and to one loop in open string theory, the D-branes do not exert any force on each other. Our examples contain pairs of non-BPS D-branes, as well as a system of BPS branes carrying different charge quantum numbers or orientations so that the combined system is not supersymmetric.

The various examples that we shall examine involve either BPS D-branes of type IIA/IIB string theories wrapped on non-supersymmetric cycles of the K3 orbifold [1, 2], or a system of BPS D-branes each wrapped on a supersymmetric cycle [3], but such that the combined system breaks all supersymmetries.1 A BPS D-brane wrapped on a supersymmetric cycle is obtained by starting with a BPS D-brane in type IIA/IIB string theory on a torus $T^{4}$, where an even number of tangential directions of the brane are along the torus. This configuration is then modded out by a $\mathbb{Z}_{2}$ transformation generated by $\mathcal{I}_{4}$, the transformation that reverses the sign of all the coordinates of $T^{4}$ [3]. On the other hand, a BPS D-brane wrapped on a non-supersymmetric cycle of K3 is obtained by starting with a non-BPS D-brane in type IIA/IIB string theory on $T^{4}$, where an odd number of tangential directions of the brane are along the torus; this is then modded out by the same $\mathbb{Z}_{2}$ transformation $\mathcal{I}_{4}$. For our analysis we shall use a T-dual description which maps the orbifold of type IIA/IIB on $T^{4}$ by $\mathcal{I}_{4}$ to the orbifold of type IIB/IIA on $T^{4}$ by $g=\mathcal{I}_{4} \cdot(-1)^{F_{L}}$, where $F_{L}$ denotes the contribution to the space-time fermion number from the left-moving sector of the world-sheet. In this description, the T-dual of a BPS D-brane wrapped on a supersymmetric cycle of K3 is obtained by taking a BPS D-brane on $T^{4}$ with an odd number of tangential directions along the torus and then modding out the theory by $g$. On the other hand the T-dual of a BPS D-brane wrapped on a non-supersymmetric cycle of K3 is obtained by taking a non-BPS D-brane with an even number of tangential directions along the torus, and then modding out the theory by $g$. In all the examples that we discuss we take $T^{4}$ to be a direct product of four circles with no background anti-symmetric tensor field.

The first example that we shall consider is that of a single non-BPS D $p$ brane of IIB/IIA situated at one of the orbifold fixed points of $T^{4} / g$, all of whose tangential directions extend along the non-compact space-time. (In the dual type IIA/IIB theory on K3 this corresponds to a BPS $(p+2)$-brane wrapped on a non-supersymmetric 2-cycle of K3.) This system is stable when the radius of each circle of the torus is larger than

\footnotetext{
${ }^{1} \mathrm{~A}$ simpler example of a bose-fermi degenerate spectrum on a non-BPS brane configuration involving

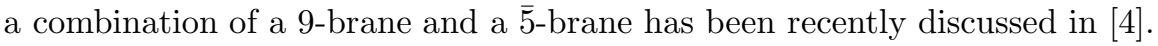


$(1 / \sqrt{2})$.2 We find that precisely when all the four radii are at their critical value, the spectrum of open strings living on the system develops exact bose-fermi degeneracy, and thus the one loop open string partition function vanishes. This implies that to this order, the force between a pair of branes of this type due to closed string exchange vanishes at all distance scales. We also find that when any of the radii is above the critical radius, the force is repulsive at all distances.

The second example involves a pair of anti-parallel BPS $\mathrm{D}(p+1)$ branes of type IIB/IIA string theory on $T^{4} / g$, where one of the tangential directions of the branes is along one of the circles of the torus, and the other tangential directions extend along the non-compact space-time. The two branes could be separated along the non-compact directions transverse to the brane, but both branes lie along a common fixed line of $T^{4} / g$. (In the dual type IIA/IIB theory on K3 orbifold, this represents a pair of $\mathrm{D}(p+2)$ branes, each wrapped on a supersymmetric 2-cycle.) This system is stable if the radius of the circle tangential to the brane is smaller than $\sqrt{2}$, and the radii of the circles transverse to the brane are each larger than $(1 / \sqrt{2})$. Again we find that precisely when all the radii take their critical values, the open string spectrum on this system develops exact bose-fermi degeneracy, even though the brane configuration is not supersymmetric.

The third example is that of a pair of non-BPS D $p$-branes of type IIB/IIA placed at two different orbifold points of $T^{4} / g$, - at diametrically opposite points of one of the circles of $T^{4}$, - with all directions tangential to the branes lying along the non-compact directions. This system is stable when the radius of this special circle is larger than $\sqrt{2}$, and the radii of the other circles are larger than $(1 / \sqrt{2})$. Again when all the radii are at their critical value, the open string spectrum on this system develops exact bose-fermi degeneracy. At the critical point there is a marginal deformation which interpolates between this system and the one in the previous example; but one can show that the bose-fermi degeneracy does not survive along this line of marginal deformation.

The fourth example is that of a pair of BPS $\mathrm{D}(p+1)$ branes in IIB/IIA on $T^{4} / g$, each with one tangential direction along a circle of the torus, but unlike the second example where these directions are anti-parallel, we take the two directions to be along two orthogonal circles of the torus representing fixed lines on $T^{4} / g$ that intersect at a fixed point. The two D-branes share the same $p+1$ non-compact tangential directions. The spectrum of open strings living on this system develops exact bose-fermi degeneracy

\footnotetext{
${ }^{2}$ We are using $\alpha^{\prime}=1$ units.
} 
when each of the two circles transverse to both the branes has radius $(1 / 2)$. This is also the critical radius below which this system of branes develops a tachyonic mode and hence becomes unstable.

There are two ways of computing the partition function of open strings living on the D-brane system, - directly from the spectrum of open strings, or using the boundary state formalism to represent the D-branes as a source for closed strings, and then computing the amplitude for emission and reabsorption of closed strings. In the open string calculation it is easy to work out the normalisation of the partition function, but sometimes it is difficult to know the rules for projection under various symmetries. In the closed string computation it is easy to find the rules for projection under various symmetries, but the computation of the normalisation factors is difficult. We use both approaches. In section 2 we use the known spectrum of open strings on BPS and non-BPS D-branes in type II string theory on $T^{4}$ modded out by $g$ to compute the partition function in examples 1 and 2 in the open string formalism. Then in section 3 we repeat the analysis for these examples in the boundary state formalism, and in the process fix the overall normalisation of the boundary states by comparing with the known answers in section 2. Then we carry out the analysis of examples 3 and 4 using the boundary state formalism.

Theories with accidental bose-fermi degeneracy have been used before [5, 6, 7, 8, 9, 10] in postulating the existence of non-supersymmetric string theories with vanishing cosmological constant. It is tempting to speculate that the type of examples described here could be useful in constructing orientifold duals of these theories.

The existence of non-BPS brane configurations which do not exert any force on each other also opens up the possibility of putting a large number of such systems together, unless higher loop corrections generate a repulsive interaction between these systems. In this case we might expect the near horizon geometry of this system to be described reliably by a solution of the supergravity equations of motion, thereby giving rise to new relations between non-supersymmetric field theories and string theory in the spirit of Refs. [11, 12, 13]. 


\section{Examples of non-BPS D-brane configurations with exact bose-fermi degeneracy}

In this section we shall construct examples of stable non-BPS brane configurations for which the spectrum of open strings living on the brane has exact bose-fermi degeneracy at all mass levels, and as a result the force between these branes due to single closed string exchange vanishes at all distances. The theory that we shall consider is type IIB/IIA string theory on $T^{4}$ modded out by $\mathcal{I}_{4} \cdot(-1)^{F_{L}}$, with $\mathcal{I}_{4}$ denoting the transformation that reverses the sign of all the coordinates of the torus and $F_{L}$ denoting the contribution to the space-time fermion number from the left-moving sector of the world-sheet. (This theory is T-dual to type IIA/IIB string theory on $T^{4} / \mathcal{I}_{4}$.) Let us denote by $x^{6}, \ldots, x^{9}$ the coordinates on $T^{4}$, by $R_{6}, \ldots, R_{9}$ the corresponding radii, and by $x^{0}, \ldots, x^{5}$ the noncompact coordinates. We shall assume that we have Dirichlet boundary conditions on the brane along the $x^{1}, x^{2}$ directions, and use a light-cone gauge formalism with $x^{1}, x^{2}$ as the light-cone directions; a standard D-brane (with Neumann boundary conditions in the time-direction) can be obtained from this by a double Wick rotation [14].

For a given configuration of D-branes, the object of interest is the open string partition function:

$$
Z=\int \frac{d t}{2 t} \operatorname{Tr}_{N S-R}\left(e^{-2 t H_{o}} \mathcal{P}\right)
$$

where NS and R denote Neveu-Schwarz and Ramond sectors, respectively, $\mathcal{P}$ is an appropriate projection operator, and $H_{o}$ is the open string Hamiltonian:

$$
H_{o}=\pi \vec{p}^{2}+\frac{1}{4 \pi} \vec{w}^{2}+\pi \sum_{\mu=0,3, \ldots 9}\left[\sum_{n=1}^{\infty} \alpha_{-n}^{\mu} \alpha_{n}^{\mu}+\sum_{r>0} r \psi_{-r}^{\mu} \psi_{r}^{\mu}\right]+\pi C_{o}
$$

Here $\vec{p}$ denotes the open string momentum along the directions for which the string has Neumann (N) boundary conditions at both ends, and $\vec{w}$ denotes the winding charge along the directions for which both ends obey Dirichlet (D) boundary conditions. $\alpha_{n}^{\mu}$ and $\psi_{r}^{\mu}$ denote respectively the bosonic and fermionic oscillators satisfying the commutation relations:

$$
\left[\alpha_{m}^{\mu}, \alpha_{n}^{\nu}\right]=m \delta^{\mu \nu} \delta_{m+n, 0}, \quad\left\{\psi_{r}^{\mu}, \psi_{s}^{\nu}\right\}=\delta^{\mu \nu} \delta_{r+s, 0} .
$$

For coordinates satisfying the same boundary condition at both ends of the open string (i.e. both Neumann $(\mathrm{N})$ or both Dirichlet (D)) $n$ always takes integer values, whereas $r$ takes integer (integer $+\frac{1}{2}$ ) values in the R (NS) sector. On the other hand, for coordinates 
satisfying different boundary conditions at the two ends of the open string (one D and one N) $n$ takes integer $+\frac{1}{2}$ values and $r$ takes integer $+\frac{1}{2}$ (integer) values in the $\mathrm{R}$ (NS)

sector. The normal ordering constant $C_{o}$ vanishes in the R-sector and is equal to $-\frac{1}{2}+\frac{s}{8}$ in the NS sector (in $\alpha^{\prime}=1$ units) where $s$ denotes the number of coordinates satisfying D-N boundary conditions. The trace, denoted by $T r$, is taken over the full Fock space of the open string, and also includes a sum (integral) over various momentum and winding numbers, and a sum over the different Chan Paton sectors.

\subsection{Example 1: BPS D-brane wrapped on non-supersymmetric cycle of K3}

We begin with a single non-BPS Dp-brane 15, 16, 17, 18] in type IIB/IIA string theory on $T^{4} /(-1)^{F_{L}} \cdot \mathcal{I}_{4}$. The D-brane is situated at one of the fixed points of $T^{4} /(-1)^{F_{L}} \cdot \mathcal{I}_{4}$, and all $p+1$ directions on the world-volume extend along the non-compact space-time. $p$ is even for type IIB string theory, and odd for type IIA string theory. (In the T-dual description this can be regarded as a BPS D- $(p+2)$-brane in IIA/IIB wrapped on a non-supersymmetric cycle of K3 [1, 2].) There are two different Chan Paton sectors, labelled by the $2 \times 2$ identity matrix $I$ and the Pauli matrix $\sigma_{1}$, -, each having its own rule for GSO $\left((-1)^{F}\right)$ projection, and $g \equiv \mathcal{I}_{4} \cdot(-1)^{F_{L}}$ projection. Thus $\mathcal{P}$ in Eq. (2.1) is given by:

$$
\mathcal{P}=\frac{1+(-1)^{F}}{2} \frac{1+g}{2} .
$$

$(-1)^{F}$ reverses the sign of all the world-sheet fermions, whereas $g$ reverses the sign of the world-sheet scalar and fermions associated with the $x^{6}, \ldots, x^{9}$ coordinates. In computing the partition function (2.1) we also need to know how $(-1)^{F}$ and $g$ act on the Fock vacuum in the different sectors; this is known [16, 17, 19. In the NS sector we have

$$
\begin{array}{rlr}
I & :(-1)^{F}=-1 & g=1 \\
\sigma_{1}: & (-1)^{F}=1 & g=-1 .
\end{array}
$$

Thus when we combine the open string spectrum from the two sectors, we see that there is no net $(-1)^{F}$ or $g$ projection, as at a given mass level we have open string states carrying both $(-1)^{F}$ charges and both $g$ charges. However, there is a net $(-1)^{F} \cdot g$ projection, since the Fock vacuum in both sectors is odd under $(-1)^{F} \cdot g$, and hence only those open string states, obtained by oscillators carrying a net $(-1)^{F} \cdot g$ charge -1 acting on the vacuum, 
are allowed in the spectrum. Thus the combined contribution of all Chan Paton factors to (2.1) from NS sector states can be written as

$$
\int \frac{d t}{2 t} \operatorname{tr}_{N S}\left(e^{-2 t H_{o}} \frac{1+(-1)^{F} \cdot g}{2}\right),
$$

where $t r$ now denotes a sum over the oscillators in a single Fock space, an integration over momenta in the non-compact directions, and a sum over winding numbers in the compact directions, but does not contain a sum over the different Chan Paton factors.

The situation in the Ramond sector is even simpler. The ground state is 16-fold degenerate due to the 8 fermionic zero modes, and it contains an equal number of states with charge 1 and -1 under each of the three operators $(-1)^{F}, g$ and $(-1)^{F} \cdot g$. Thus $\operatorname{Tr}_{R}\left(e^{-2 t H_{o}}(-1)^{F}\right), \operatorname{Tr}_{R}\left(e^{-2 t H_{o}} g\right)$, and $\operatorname{Tr}_{R}\left(e^{-2 t H_{o}}(-1)^{F} \cdot g\right)$ all vanish, and when we combine the spectrum from the two Chan Paton sectors, the contribution to (2.1) can be written as

$$
\int \frac{d t}{2 t} \frac{1}{2} \operatorname{tr}_{R}\left(e^{-2 t H_{o}}\right)
$$

We can now evaluate the contribution from each sector separately. We get

$$
\operatorname{tr}_{N S}\left(e^{-2 t H_{o}}\right)=\frac{A}{(2 \pi)^{p+1}}(2 t)^{-\frac{p+1}{2}} \frac{f_{3}(\tilde{q})^{8}}{f_{1}(\tilde{q})^{8}}\left(\prod_{i=6}^{9} \sum_{n_{i} \in \mathbb{Z}} \tilde{q}^{2 R_{i}^{2} n_{i}^{2}}\right)
$$

where $A$ is the (infinite) $(p+1)$-dimensional volume of the brane in the non-compact directions,

$$
\tilde{q}=e^{-\pi t}
$$

and $f_{i}$ are defined in the usual manner [20]:

$$
\begin{aligned}
& f_{1}(\tilde{q})=\tilde{q}^{\frac{1}{12}} \prod_{n=1}^{\infty}\left(1-\tilde{q}^{2 n}\right) \\
& f_{2}(\tilde{q})=\sqrt{2} \tilde{q}^{\frac{1}{12}} \prod_{n=1}^{\infty}\left(1+\tilde{q}^{2 n}\right) \\
& f_{3}(\tilde{q})=\tilde{q}^{-\frac{1}{24}} \prod_{n=1}^{\infty}\left(1+\tilde{q}^{2 n-1}\right), \\
& f_{4}(\tilde{q})=\tilde{q}^{-\frac{1}{24}} \prod_{n=1}^{\infty}\left(1-\tilde{q}^{2 n-1}\right) .
\end{aligned}
$$

The origin of the various factors in (2.8) is as follows. The $A(2 \pi)^{-p+1}(2 t)^{-(p+1) / 2}$ factor comes from integration over the open string momenta along the non-compact directions 
and the $f_{3}(\tilde{q})^{8} / f_{1}(\tilde{q})^{8}$ factor represents the contribution of the bosonic and the fermionic oscillators. The last factor $\prod_{i} \sum_{n_{i}} \tilde{q}^{2 R_{i}^{2} n_{i}^{2}}$ comes from the sum over open string winding modes along the compact directions.

Similarly we get

$$
\operatorname{tr}_{N S}\left(e^{-2 t H_{o}}(-1)^{F} \cdot g\right)=-\frac{A}{(2 \pi)^{p+1}}(2 t)^{-\frac{p+1}{2}} \cdot 4 \cdot \frac{f_{3}(\tilde{q})^{4} f_{4}(\tilde{q})^{4}}{f_{1}(\tilde{q})^{4} f_{2}(\tilde{q})^{4}} .
$$

The contribution from the momentum integration along the non-compact directions remains the same but the oscillator contribution changes, since four of the bosonic oscillators and four of the fermionic oscillators change sign under $(-1)^{F} \cdot g$. There is no contribution from the winding sector, since $(-1)^{F} \cdot g$ takes a state with winding charge $\vec{w}$ to a state with winding charge $-\vec{w}$, and hence the contribution from these states vanishes in the trace. Finally, the overall - sign reflects the fact that the NS sector ground state is odd under $(-1)^{F} \cdot g$ (which is the reason why this model is free of tachyons).

The contribution from the Ramond sector can also be evaluated in a straightforward manner. We get

$$
\operatorname{tr}_{R}\left(e^{-2 t H_{o}}\right)=\frac{A}{(2 \pi)^{p+1}}(2 t)^{-\frac{p+1}{2}} \frac{f_{2}(\tilde{q})^{8}}{f_{1}(\tilde{q})^{8}}\left(\prod_{i=6}^{9} \sum_{n_{i} \in Z} \tilde{q}^{2 R_{i}^{2} n_{i}^{2}}\right) .
$$

Comparing with Eq. (2.8) we see that the contribution from momentum integration along the non-compact directions, and the sum over winding modes in the compact direction remains the same. The only change is in the contribution from the fermionic oscillators.

Combining the contribution from all sectors, and using the (abstruse) identity

$$
f_{3}(\widetilde{q})^{8}-f_{2}(\widetilde{q})^{8}=f_{4}(\widetilde{q})^{8},
$$

we see that the total partition function is given by:

$$
Z=\frac{1}{2} \int \frac{d t}{2 t} \frac{A}{(2 \pi)^{p+1}}(2 t)^{-\frac{p+1}{2}}\left[\frac{f_{4}(\tilde{q})^{8}}{f_{1}(\tilde{q})^{8}}\left(\prod_{i=6}^{9} \sum_{n_{i} \in \mathbb{Z}} \tilde{q}^{2 R_{i}^{2} n_{i}^{2}}\right)-4 \cdot \frac{f_{3}(\tilde{q})^{4} f_{4}(\tilde{q})^{4}}{f_{1}(\tilde{q})^{4} f_{2}(\tilde{q})^{4}}\right] .
$$

Let us now consider the case where $R_{i}=\frac{1}{\sqrt{2}}$ for each $i$. In this case we get

$$
\sum_{n_{i} \in \mathbb{Z}} \tilde{q}^{2 R_{i}^{2} n_{i}^{2}}=\sum_{n \in \mathbb{Z}} \tilde{q}^{n^{2}}
$$

Using the sum and the product representation of the Jacobi $\vartheta$-function $\vartheta_{3}(0 \mid \tau)$ [21],

$$
\vartheta_{3}(0 \mid \tau)=\sum_{n \in \mathbb{Z}} \tilde{q}^{n^{2}}=\prod_{n=1}^{\infty}\left(1-\tilde{q}^{2 n}\right)\left(1+\tilde{q}^{2 n-1}\right)^{2}=f_{1}(\tilde{q}) f_{3}^{2}(\tilde{q}),
$$


where $\tilde{q}=e^{2 \pi i \tau}$, and the identity

$$
f_{4}(\tilde{q}) \frac{1}{\sqrt{2}} f_{2}(\tilde{q}) f_{3}(\tilde{q})=1
$$

we get

$$
\sum_{n \in \mathbb{Z}} \tilde{q}^{n^{2}}=\sqrt{2} \frac{f_{1}(\tilde{q}) f_{3}(\tilde{q})}{f_{2}(\tilde{q}) f_{4}(\tilde{q})} .
$$

Using Eqs. (2.15) and (2.18), (2.14) then becomes

$$
Z=0
$$

Since the integrand of $Z$ vanishes for all $t$, this shows that there is exact degeneracy between bosonic and fermionic open string states at all mass level, although the brane is non-BPS. In order to find the bosonic and the fermionic spectrum separately, we need to evaluate (2.12) or the sum of (2.8) and (2.11). This is given by:

$$
\frac{A}{(2 \pi)^{p+1}}(2 t)^{-\frac{p+1}{2}} 4 \cdot \frac{f_{2}(\tilde{q})^{4} f_{3}(\tilde{q})^{4}}{f_{1}(\tilde{q})^{4} f_{4}(\tilde{q})^{4}} .
$$

This is proportional to the partition function of bosonic (or fermionic) open string states stretched between a BPS $p$-brane and a BPS $(p+4)$-brane in type II string theory.

Note that the critical radii where the spectrum of open strings develops exact bosefermi degeneracy correspond precisely to the values below which the non-BPS D-brane becomes unstable against decay into a pair of BPS branes [15]. This is not a coincidence. For $R_{i}>\frac{1}{\sqrt{2}}$ the massless spectrum contains four bosonic states from sector $I$, four fermionic states from sector $I$, and four fermionic states from sector $\sigma_{1}$. In order to have bose-fermi degeneracy at the massless level, we need four extra massless bosonic states which are provided by some of the modes from sector $\sigma_{1}$ becoming massless at the critical radius. As we decrease any of the $R_{i}$ below the critical radius, the corresponding mode becomes tachyonic signalling an instability in the system.

We can use this result to conclude that when $R_{6}=R_{7}=R_{8}=R_{9}=\frac{1}{\sqrt{2}}$, the force between a pair of non-BPS branes of this kind vanishes at all distances. To see this we note that if we consider a pair of such branes separated by a distance $r$ in any of the non-compact directions transverse to the brane, then the partition function of open strings stretched from one of the branes to another is given by the same expression as (2.14) except for an overall extra factor of $\tilde{q}^{r^{2} / 2 \pi^{2}}$ in the integrand, reflecting the energy 
associated with the tension of the open string stretched over a distance $r$. Thus at the critical radius the partition function vanishes, reflecting that the potential energy $V(r)$ between the pair of branes (which is equal to negative of the partition function) vanishes identically for all $r$.

Since $\sum_{n_{i} \in \mathbb{Z}} \tilde{q}^{2 R_{i}^{2} n_{i}^{2}}$ is a monotonically decreasing function of $R_{i}$ (as $0<\tilde{q}<1$ ), we see

that for $R_{i}>\frac{1}{\sqrt{2}}$ the integrand of Eq. (2.14) is a negative definite function. Thus $V(r)$ is positive definite. Furthermore since $V(r)$ only depends on $r$ via $\tilde{q}^{r^{2} / 2 \pi^{2}}$, it follows by the same argument that $V^{\prime}(r)$ is negative, and hence that $V(r)$ is a monotonically decreasing function of $r$. Thus for $R_{i}>\frac{1}{\sqrt{2}}$, where the non-BPS brane is stable, the interaction between a pair of such branes is repulsive at all distances.

\subsection{Example 2: Pair of BPS D-branes wrapped on supersym- metric cycles of $\mathrm{K} 3$}

In this section we shall study the example of a parallel BPS D- $(p+1)$-brane $\overline{\mathrm{D}}-(p+1)$ brane pair, where one direction extends along $x^{9}$, and the other $p+1$ directions on the worldvolume lie along the non-compact space-time directions. As before we are considering type IIB/IIA string theory on $T^{4}$ modded out by $(-1)^{F_{L}} \cdot \mathcal{I}_{4}$, where $\mathcal{I}_{4}$ inverts $x^{6}, \ldots, x^{9}$. Consistency requires that $p$ is odd for type IIA string theory, and even for type IIB string theory. We consider the situation where we do not have a Wilson line on any of the branes, and we take them both to be situated at $x^{6}=x^{7}=x^{8}=0$, while they can be separated by a distance $r$ along any of the non-compact directions transverse to the brane. There are two different cases, depending on whether the brane and the anti-brane carry the same or opposite twisted sector RR charge [15]; here we shall only consider the situation where the twisted sector RR charge is the same for both branes. (In the T-dual description this corresponds to a pair of fractional $p$-branes situated at one of the orbifold fixed points of $T^{4} / \mathcal{I}_{4}$ in type IIA/IIB string theory on $T^{4} / \mathcal{I}_{4}$, carrying the same twisted sector RR charge, but opposite untwisted sector RR charge. Each of these can be interpreted as a BPS $(p+2)$-brane wrapped on a supersymmetric cycle [3]. Thus each system is individually BPS, but the combined system is not BPS, as the RR charges of the two systems are not aligned.)

We shall compute the partition function of open strings living on this system. Since each of the branes is individually BPS, the spectrum of open strings with both ends living on the same brane is automatically supersymmetric. Thus we only need to focus on the 
open strings with one end on the D-brane and the other end on the $\bar{D}$-brane. There are two such sectors, related by reversal of the orientation of the open strings. But since both sectors contribute equally to the partition function we shall restrict our attention to only one sector. Thus there is no sum over Chan Paton factors, and we can express the partition function (2.1) as

$$
Z=\int \frac{d t}{2 t} \operatorname{tr}_{N S-R}\left(e^{-2 t H_{o}} \frac{1+(-1)^{F}}{2} \frac{1+g}{2}\right) .
$$

The action of $(-1)^{F}$ and $g$ on the world-sheet fields is identical to that described in the last example, but we need to know the action on the vacuum. Since the open string is stretched between the D-string and the $\bar{D}$-string, the NS sector ground state is $(-1)^{F}$ even. On the other hand, from the analysis of Ref. [15] we know that the NS sector ground state carrying zero momentum is projected out under the $g$ projection. Thus it is odd under $g$. In the Ramond sector we have a 16-fold degeneracy, and as in the previous example, the ground state contains an equal number of states carrying +1 and -1 eigenvalues of $(-1)^{F}, g$ and $(-1)^{F} \cdot g$. Thus only the $\operatorname{tr}_{R}\left(e^{-2 t H_{o}}\right)$ term contributes from this sector.

We can now easily write down the expression for the contribution from the various sectors,

$$
\operatorname{tr}_{N S}\left(e^{-2 t H_{o}}\right)=\frac{A}{(2 \pi)^{p+1}}(2 t)^{-\frac{p+1}{2}} \tilde{q}^{r^{2} / 2 \pi^{2}} \frac{f_{3}(\tilde{q})^{8}}{f_{1}(\tilde{q})^{8}}\left(\prod_{i=6}^{8} \sum_{n_{i} \in \mathbb{Z}} \tilde{q}^{2 R_{i}^{2} n_{i}^{2}}\right)\left(\sum_{m_{9} \in \mathbb{Z}} \tilde{q}^{2 m_{9}^{2} / R_{9}^{2}}\right) .
$$

Compared to (2.8), there is an extra factor of $\tilde{q}^{r^{2} / 2 \pi^{2}}$ reflecting the effect of the transverse separation between the two branes, and the sum over winding numbers along the 9th direction has been replaced by a sum over momenta, since we now have a Neumann boundary condition along this direction. Similarly we get

$$
\operatorname{tr}_{N S}\left(e^{-2 t H_{o}}(-1)^{F}\right)=\frac{A}{(2 \pi)^{p+1}}(2 t)^{-\frac{p+1}{2}} \tilde{q}^{r^{2} / 2 \pi^{2}} \frac{f_{4}(\tilde{q})^{8}}{f_{1}(\tilde{q})^{8}}\left(\prod_{i=6}^{8} \sum_{n_{i} \in \mathbb{Z}} \tilde{q}^{2 R_{i}^{2} n_{i}^{2}}\right)\left(\sum_{m_{9} \in \mathbb{Z}} \tilde{q}^{2 m_{9}^{2} / R_{9}^{2}}\right) .
$$

\footnotetext{
${ }^{3}$ We are using a slightly different convention from the one used in [15]. There the action of $g$ and $(-1)^{F}$ on the NS sector ground state was defined to be the same as in the case of open strings with both ends on the D-brane, but the sign of $(-1)^{F}$ and $g$ in the projection operator was chosen to be negative. Here we have chosen the signs of $(-1)^{F}$ and $g$ in the projection operator to be positive, but are taking the action of these operators on the NS sector ground state to be opposite of that for open strings with both ends on the D-brane.
} 


$$
\begin{gathered}
\operatorname{tr}_{N S}\left(e^{-2 t H_{o}} g\right)=-\frac{A}{(2 \pi)^{p+1}}(2 t)^{-\frac{p+1}{2}} \tilde{q}^{r^{2} / 2 \pi^{2}} \cdot 4 \cdot \frac{f_{3}(\tilde{q})^{4} f_{4}(\tilde{q})^{4}}{f_{1}(\tilde{q})^{4} f_{2}(\tilde{q})^{4}} . \\
\operatorname{tr}_{N S}\left(e^{-2 t H_{o}}(-1)^{F} \cdot g\right)=-\frac{A}{(2 \pi)^{p+1}}(2 t)^{-\frac{p+1}{2}} \tilde{q}^{r^{2} / 2 \pi^{2}} \cdot 4 \cdot \frac{f_{3}(\tilde{q})^{4} f_{4}(\tilde{q})^{4}}{f_{1}(\tilde{q})^{4} f_{2}(\tilde{q})^{4}} . \\
\operatorname{tr}_{R}\left(e^{-2 t H_{o}}\right)=\frac{A}{(2 \pi)^{p+1}}(2 t)^{-\frac{p+1}{2}} \tilde{q}^{r^{2} / 2 \pi^{2}} \frac{f_{2}(\tilde{q})^{8}}{f_{1}(\tilde{q})^{8}}\left(\prod_{i=6}^{8} \sum_{n_{i} \in \mathbb{Z}} \tilde{q}^{2 R_{i}^{2} n_{i}^{2}}\right)\left(\sum_{m_{9} \in \mathbb{Z}} \tilde{q}^{2 m_{9}^{2} / R_{9}^{2}}\right) .
\end{gathered}
$$

Substituting these into (2.21) and using the identity (2.13), $Z$ becomes in this case

$$
\int \frac{d t}{2 t} \frac{1}{2} \frac{A}{(2 \pi)^{p+1}}(2 t)^{-\frac{p+1}{2}} \tilde{q}^{r^{2} / 2 \pi^{2}}\left[\frac{f_{4}(\tilde{q})^{8}}{f_{1}(\tilde{q})^{8}}\left(\prod_{i=6}^{8} \sum_{n_{i} \in \mathbb{Z}} \tilde{q}^{2 R_{i}^{2} n_{i}^{2}}\right)\left(\sum_{m_{9} \in \mathbb{Z}} \tilde{q}^{2 m_{9}^{2} / R_{9}^{2}}\right)-4 \cdot \frac{f_{3}(\tilde{q})^{4} f_{4}(\tilde{q})^{4}}{f_{1}(\tilde{q})^{4} f_{2}(\tilde{q})^{4}}\right] .
$$

Now we note that for $R_{6}=R_{7}=R_{8}=\frac{1}{\sqrt{2}}$ and $R_{9}=\sqrt{2}$,

$$
\left(\prod_{i=6}^{8} \sum_{n_{i} \in \mathbb{Z}} \tilde{q}^{2 R_{i}^{2} n_{i}^{2}}\right)\left(\sum_{m_{9} \in \mathbb{Z}} \tilde{q}^{2 m_{9}^{2} / R_{9}^{2}}\right)=\left(\sum_{n \in \mathbb{Z}} \tilde{q}^{n^{2}}\right)^{4}=\left(\sqrt{2} \frac{f_{1}(\tilde{q}) f_{3}(\tilde{q})}{f_{2}(\tilde{q}) f_{4}(\tilde{q})}\right)^{4} .
$$

Substituting this into Eq. (2.27) we see that

$$
Z=0
$$

Thus the spectrum of open strings has exact bose-fermi degeneracy even though the brane configuration is not supersymmetric. At this critical point in the moduli space, the force between the pair of branes vanishes for all separations.

By repeating the analysis of the previous example we can easily verify that the partition function in the NS or R sector is again proportional to that of a BPS D- $p-\mathrm{D}-(p+4)$ brane system in type II string theory. Also, if $R_{i}>\frac{1}{\sqrt{2}}$ for $6 \leq i \leq 8$, and $R_{9}<\sqrt{2}$, the interaction between the pair of branes is repulsive at all distances.

\section{The boundary state approach}

It is instructive to compare the above derivation of the bose-fermi degeneracy of the open string spectrum with that using the closed string theory point of view, describing Dirichlet branes as boundary states [20, 22, 23, 24, 25, 26]. We shall use the conventions of [15] in the following. Let us first treat the two cases that we have analysed above in turn in order to fix the relevant normalisation constants of the boundary states; we shall then also construct two new examples. 


\subsection{Example 1: BPS D-brane wrapped on non-supersymmetric cycle of K3}

This is the same example that we studied in section 2.1, - namely, a non-BPS $p$-brane of IIB/IIA on $T^{4} /(-1)^{F_{L}} \cdot \mathcal{I}_{4}$, all of whose tangential directions lie along the non-compact space-time. Let us denote by $\mathcal{C} \subset\{0,3,4,5\}$ the set of $p+1$ indices for which the Dirichlet brane satisfies Neumann boundary conditions, and denote by $\mathcal{D}$ the complement of $\mathcal{C}$ in $\{0,3,4,5\}$. We also denote by $\mathcal{D}_{c}$ the union of $\mathcal{D}$ with $\{1,2\}$, and by $\hat{\mathcal{D}}$ the union of $\mathcal{D}$ with $\{6,7,8,9\}$. The boundary state that represents the non-BPS $p$-brane is of the form [16]

$$
\begin{aligned}
|\widetilde{D} p, \mathbf{a}, \mathbf{b}, \epsilon\rangle=\frac{1}{2}( & \left.|B p, \mathbf{a}, \mathbf{b},+\rangle_{N S N S ; U}-|B p, \mathbf{a}, \mathbf{b},-\rangle_{N S N S ; U}\right) \\
& +\frac{\epsilon}{2}\left(|B p, \mathbf{a}, \mathbf{b},+\rangle_{R R ; T}+|B p, \mathbf{a}, \mathbf{b},-\rangle_{R R ; T}\right)
\end{aligned}
$$

where the first two (last two) states are coherent states in the untwisted NSNS (twisted $\mathrm{RR}$ ) sector. a denotes the location of the brane in the non-compact directions with $a^{1}=a^{2}=0$, and $\mathbf{b}$ denotes the location of the brane in the compact directions. Since we always take the brane to lie at one of the fixed points of $T^{4} / g, b^{i}$ can only take the values 0 or $\pi R_{i}(6 \leq i \leq 9)$. $\epsilon$ can take values \pm 1 , and denotes the sign of the twisted sector $\mathrm{RR}$ charge carried by the brane. Up to an overall normalisation, the coherent states are (uniquely) characterised by the conditions

$$
\begin{aligned}
& X^{\mu}(\tau=0, \sigma)|B p, \mathbf{a}, \mathbf{b}, \eta\rangle=a^{\mu} \quad \text { for } \quad \mu \in \mathcal{D} \\
& X^{\mu}(\tau=0, \sigma)|B p, \mathbf{a}, \mathbf{b}, \eta\rangle=b^{\mu} \quad \text { for } \quad 6 \leq \mu \leq 9 \\
& \partial_{\tau} X^{\mu}(\tau=0, \sigma)|B p, \mathbf{a}, \mathbf{b}, \eta\rangle=0 \quad \text { for } \quad \mu \in \mathcal{C} \\
& \left(\psi^{\mu}(\tau=0, \sigma)-i \eta \tilde{\psi}^{\mu}(\tau=0, \sigma)\right)|B p, \mathbf{a}, \mathbf{b}, \eta\rangle=0 \quad \text { for } \quad \mu \in \hat{\mathcal{D}} \\
& \left(\psi^{\mu}(\tau=0, \sigma)+i \eta \tilde{\psi}^{\mu}(\tau=0, \sigma)\right)|B p, \mathbf{a}, \mathbf{b}, \eta\rangle=0 \quad \text { for } \quad \mu \in \mathcal{C} \\
& x^{\mu}|B p, \mathbf{a}, \mathbf{b}, \eta\rangle=0 \quad \text { for } \quad \mu=1,2 \text {. }
\end{aligned}
$$

Here $X^{\mu}$ denotes the bosonic coordinate field, $\psi^{\mu}$ and $\tilde{\psi}^{\mu}$ its right- and left-moving superpartner on the closed string world-sheet, and $x^{\mu}$ the zero mode of $X^{\mu}$. $\eta$ can take values \pm 1 . More explicitly the boundary state $|B p, \mathbf{a}, \mathbf{b}, \eta\rangle$ can be written as

$$
|B p, \mathbf{a}, \mathbf{b}, \eta\rangle_{N S N S ; U}=\mathcal{N} \int\left(\prod_{\mu \in \mathcal{D}_{c}} d k^{\mu} e^{i k \cdot \mathbf{a}}\right)\left(\prod_{i=6}^{9} \sum_{m_{i} \in \mathbb{Z}} e^{i m_{i} b^{i} / R_{i}}\right)|B p, \widehat{\mathbf{k}, \mathbf{m}}, \eta\rangle_{N S N S ; U}
$$

\footnotetext{
${ }^{4}$ We shall be using the convention that $\widetilde{D}$ denotes a non-BPS D-brane, and $D$ denotes a BPS D-brane.
} 
and

$$
|B p, \mathbf{a}, \mathbf{b}, \eta\rangle_{R R ; T}=2 i \widetilde{\mathcal{N}} \int\left(\prod_{\mu \in \mathcal{D}_{c}} d k^{\mu} e^{i \mathbf{k} \cdot \mathbf{a}}\right)|B p, \widehat{\mathbf{k}, \mathbf{b}}, \eta\rangle_{R R ; T}
$$

where $\mathbf{k}$ denotes the momentum in the non-compact directions, and $m_{i} / R_{i}$ the momentum along the $i$ th compact direction. $|B p, \widehat{\cdots}, \eta\rangle$ is the coherent momentum eigenstate

$$
\begin{aligned}
|B p, \widehat{\cdots}, \eta\rangle=\exp \left(\sum_{n=1}^{\infty}\right. & {\left[-\frac{1}{n} \sum_{\mu \in \mathcal{C}} \alpha_{-n}^{\mu} \tilde{\alpha}_{-n}^{\mu}+\frac{1}{n} \sum_{\mu \in \hat{\mathcal{D}}} \alpha_{-n}^{\mu} \tilde{\alpha}_{-n}^{\mu}\right] } \\
& \left.+i \eta \sum_{r>0}\left[-\sum_{\mu \in \mathcal{C}} \psi_{-r}^{\mu} \tilde{\psi}_{-r}^{\mu}+\sum_{\mu \in \mathcal{\mathcal { D }}} \psi_{-r}^{\mu} \tilde{\psi}_{-r}^{\mu}\right]\right)|\cdots, \eta\rangle^{(0)} .
\end{aligned}
$$

Here $\alpha_{n}^{\mu}, \tilde{\alpha}_{n}^{\mu}$ are the right- and left-moving modes of $X^{\mu}$, and $\psi_{r}^{\mu}$ and $\tilde{\psi}_{r}^{\mu}$ are the modes of $\psi^{\mu}$ and $\tilde{\psi}^{\mu}$, respectively. $n$ is integer in the untwisted NSNS sector, and in the twisted $\mathrm{RR}$ sector for $\mu=0,3,4,5$, and half-integer in the twisted RR sector for $\mu=6,7,8,9 . r$ is half-integer in the untwisted NSNS sector, and in the twisted RR sector for $\mu=6,7,8,9$, and integer in the twisted RR sector for the other values of $\mu .|\cdots, \eta\rangle^{(0)}$ denotes the Fock vacuum labelled by the quantum numbers $\cdots$. In the untwisted sector $\cdots$ correspond to the quantum numbers $\{\mathbf{k}, \mathbf{m}\}$, whereas in the twisted sector $\cdots$ stand for the quantum numbers $\{\mathbf{k}, \mathbf{b}\}$. The Fock vacuum in the twisted $R R$ sector is degenerate, but the state appearing in (3.5) is uniquely determined by the condition coming from the fermionic zero modes in Eq. (3.2). $\mathcal{N}, \widetilde{\mathcal{N}}$ are normalisation factors to be determined later.

We are interested in the tree level amplitude that describes the exchange of closed string states between two identical non-BPS Dp-branes which are located at the same fixed point of $T^{4} / g$ (at $\mathbf{b}=0$, say) and whose positions along the non-compact directions (indexed by $\mathcal{D}_{c}$ ) are described by $\mathbf{a}_{1}$ and $\mathbf{a}_{2}$. . This amplitude is given by

$$
\int_{0}^{\infty} d l\left\langle\widetilde{D} p, \mathbf{a}_{1}, \mathbf{b}=0, \epsilon\left|e^{-l H_{c}}\right| \widetilde{D} p, \mathbf{a}_{2}, \mathbf{b}=0, \epsilon\right\rangle,
$$

where $H_{c}$ is the closed string hamiltonian in light cone gauge,

$$
H_{c}=\pi \vec{p}^{2}+\frac{1}{4 \pi} \vec{w}^{2}+2 \pi \sum_{\mu=0,3, \ldots, 9}\left[\sum_{n=1}^{\infty}\left(\alpha_{-n}^{\mu} \alpha_{n}^{\mu}+\tilde{\alpha}_{-n}^{\mu} \tilde{\alpha}_{n}^{\mu}\right)+\sum_{r>0}\left(\psi_{-r}^{\mu} \psi_{r}^{\mu}+\tilde{\psi}_{-r}^{\mu} \tilde{\psi}_{r}^{\mu}\right)\right]+2 \pi C_{c} .
$$

\footnotetext{
${ }^{5}$ In order to compute the self-interaction of such a non-BPS D-brane we simply need to take $\mathbf{a}_{1}=\mathbf{a}_{2}$.
} 
The constant $C_{c}$ takes the value -1 in the untwisted NSNS sector, and 0 in the untwisted RR, twisted NSNS, and twisted RR sectors. $\vec{p}$ and $\vec{w}$ denote the momentum and winding charges as usual. Using standard techniques this amplitude can be determined to be

$$
\frac{1}{2} \int_{0}^{\infty} d l l^{-\frac{5-p}{2}} e^{-\frac{\left(\mathbf{a}_{1}-\mathbf{a}_{2}\right)^{2}}{4 \pi l}}\left[\mathcal{N}^{2}\left(\prod_{i=6}^{9} \sum_{m_{i} \in \mathbb{Z}} e^{-l \pi m_{i}^{2} / R_{i}^{2}}\right) \frac{f_{3}^{8}(q)-f_{4}^{8}(q)}{f_{1}^{8}(q)}-\widetilde{\mathcal{N}}^{2} \frac{f_{2}^{4}(q) f_{3}^{4}(q)}{f_{1}^{4}(q) f_{4}^{4}(q)}\right],
$$

where $q=e^{-2 \pi l}$. In order to determine the normalisation constants, we apply a modular transformation, setting $t=1 / 2 l$, which converts the closed string tree amplitude into an open string loop amplitude. In the present case, using the transformation properties of the $f_{i}$ functions,

$$
\begin{array}{lll}
f_{1}\left(e^{-\pi / t}\right)=\sqrt{t} f_{1}\left(e^{-\pi t}\right), & f_{2}\left(e^{-\pi / t}\right)=f_{4}\left(e^{-\pi t}\right), \\
f_{3}\left(e^{-\pi / t}\right)=f_{3}\left(e^{-\pi t}\right), & f_{4}\left(e^{-\pi / t}\right) & =f_{2}\left(e^{-\pi t}\right),
\end{array}
$$

together with the identity

$$
\sum_{m \in \mathbb{Z}} e^{-\pi l(m / R)^{2}}=\frac{R}{\sqrt{l}} \sum_{n \in \mathbb{Z}} e^{-2 t \pi(n R)^{2}}
$$

we can express $(3.8)$ as

$$
\begin{aligned}
\frac{1}{2} \int_{0}^{\infty} \frac{d t}{2 t} t^{-\frac{p+1}{2}} \tilde{q}^{\frac{\left(\mathbf{a}_{1}-\mathbf{a}_{2}\right)^{2}}{2 \pi^{2}}} 2^{\frac{5-p}{2}}\left[4 \mathcal{N}^{2}\left(\prod_{j=6}^{9} R_{j}\right)\left(\prod_{i=6}^{9} \sum_{n_{i} \in \mathbb{Z}} \tilde{q}^{2 n_{i}^{2} R_{i}^{2}}\right) \frac{f_{3}^{8}(\tilde{q})-f_{2}^{8}(\tilde{q})}{f_{1}^{8}(\tilde{q})}\right. \\
\left.-\widetilde{\mathcal{N}}^{2} \frac{f_{4}^{4}(\tilde{q}) f_{3}^{4}(\tilde{q})}{f_{1}^{4}(\tilde{q}) f_{2}^{4}(\tilde{q})}\right]
\end{aligned}
$$

where $\tilde{q}=e^{-\pi t}$. This agrees with the result of the open string calculation (2.14) provided that

$$
32 R_{6} R_{7} R_{8} R_{9} \mathcal{N}^{2}=\frac{A}{(2 \pi)^{p+1}} \quad 2 \widetilde{\mathcal{N}}^{2}=\frac{A}{(2 \pi)^{p+1}} .
$$

Using these values of $\mathcal{N}$ and $\widetilde{\mathcal{N}},(3.8)$ reduces to

$$
\begin{gathered}
\frac{A}{(2 \pi)^{p+1}} \frac{1}{4} \int_{0}^{\infty} d l l^{-\frac{5-p}{2}} e^{-\frac{\left(\mathbf{a}_{1}-\mathbf{a}_{2}\right)^{2}}{4 \pi l}}\left[\frac{1}{16 R_{6} R_{7} R_{8} R_{9}}\left(\prod_{i=6}^{9} \sum_{m_{i} \in \mathbb{Z}} e^{-l \pi m_{i}^{2} / R_{i}^{2}}\right) \frac{f_{3}^{8}(q)-f_{4}^{8}(q)}{f_{1}^{8}(q)}\right. \\
\left.-\frac{f_{2}^{4}(q) f_{3}^{4}(q)}{f_{1}^{4}(q) f_{4}^{4}(q)}\right] .
\end{gathered}
$$

At the critical radii $R_{i}=\frac{1}{\sqrt{2}}$ for $i=6,7,8,9$, the sums over $m_{i}$ can be simplified using (2.18), and together with (2.13) this implies that the integrand in (3.13) vanishes identically. 
From the point of view of the closed string calculation, the first line in (3.13) contains the attractive (bulk) gravitational force that is mediated by the massless fields from the untwisted NSNS sector, whereas the second line contains the repulsive force between objects carrying the same charge with respect to the twisted RR sector. The latter is independent of the radii of the transverse directions, but the former is inversely proportional to the radii, and also contains winding contributions which become less and less relevant as the radii are increased. Indeed, in the uncompactified theory, the untwisted sector contribution to the integrand would be proportional to $l^{-(9-p) / 2}$ for large $l$, indicating that the gravitational attraction is much weaker at long distances than the repulsive force due to the charges.

\subsection{Example 2: Pair of BPS D-branes wrapped on supersym- metric cycles of $\mathrm{K} 3$}

This is the same example discussed in section 2.2. In this case we are interested in a system containing a BPS D- $(p+1)$-brane and its anti-brane in type IIB/IIA on $T^{4}$ modded out by $\mathcal{I}_{4} \cdot(-1)^{F_{L}}$. The branes stretch along the $x^{9}$ direction, and the other $p+1$ directions of their world-volumes are in the non-compact directions. (In particular, they lie in the subspace $x^{i}=b^{i}$ for $6 \leq i \leq 8$ where $b^{i}$ can take values 0 or $\pi R_{i}$.) None of the branes carry any Wilson line. If a denotes the location of such a brane in the non-compact directions $\left(\subset\left(x^{1}, \ldots, x^{5}\right)\right)$ transverse to the brane, the boundary state describing this BPS D- $(p+1)$ brane is of the form 15.

$$
\begin{aligned}
|D(p+1), \mathbf{a}, \mathbf{b}, \epsilon, \kappa\rangle=\frac{1}{2}( & \left.|B(p+1), \mathbf{a}, \mathbf{b}\rangle_{N S N S ; U}+\epsilon|B(p+1), \mathbf{a}, \mathbf{b}\rangle_{R R ; U}\right) \\
& +\frac{1}{2 \sqrt{2}} \epsilon \kappa\left(|B(p+1), \mathbf{a}, \mathbf{b} ; 0\rangle_{N S N S ; T}+\epsilon|B(p+1), \mathbf{a}, \mathbf{b} ; 0\rangle_{R R ; T}\right) \\
& +\frac{1}{2 \sqrt{2}} \epsilon \kappa\left(\left|B(p+1), \mathbf{a}, \mathbf{b} ; \pi R_{9}\right\rangle_{N S N S ; T}\right. \\
& \left.+\epsilon\left|B(p+1), \mathbf{a}, \mathbf{b} ; \pi R_{9}\right\rangle_{R R ; T}\right) .
\end{aligned}
$$

$\epsilon$ and $\kappa$ can take values \pm 1 and denote the sign of the untwisted sector $R R$ charge and the twisted sector RR charge, respectively. (Thus $\epsilon= \pm$ corresponds to the brane and the anti-brane, respectively.) The suffices indicate the closed string sector to which each state belongs. For the case of the twisted sectors, NSNS;T and RR;T, we have also indicated 
whether the state is in the sector localised at the fixed point $x^{6}=x^{7}=x^{8}=x^{9}=0$ of $T^{4} / g$ (as is the case for the states in the second line of Eq. (3.14)) or at the fixed point $x^{6}=x^{7}=x^{8}=0, x^{9}=\pi R_{9}$ (as is the case for the states in the third and fourth line of Eq. (3.14)). Note that the sign of the twisted sector charges at the $x^{9}=0$ and the $x^{9}=\pi R_{9}$ ends are taken to be the same. This is a consequence of the fact that the branes do not carry any Wilson line along $x^{9}$; more general cases have been discussed in Ref. [15]. The different boundary states appearing on the right hand side of Eqs. (3.14) are given as

$$
\begin{aligned}
& |B(p+1), \mathbf{a}, \mathbf{b}\rangle_{N S N S ; U}=\frac{1}{\sqrt{2}}\left(|B(p+1), \mathbf{a}, \mathbf{b},+\rangle_{N S N S ; U}\right. \\
& \left.-|B(p+1), \mathbf{a}, \mathbf{b},-\rangle_{N S N S ; U}\right) \\
& |B(p+1), \mathbf{a}, \mathbf{b}\rangle_{R R ; U}=\frac{1}{\sqrt{2}}\left(|B(p+1), \mathbf{a}, \mathbf{b},+\rangle_{R R ; U}+|B(p+1), \mathbf{a}, \mathbf{b},-\rangle_{R R ; U}\right) \\
& \left|B(p+1), \mathbf{a}, \mathbf{b} ; c^{9}\right\rangle_{N S N S ; T}=\frac{1}{\sqrt{2}}\left(\left|B(p+1), \mathbf{a}, \mathbf{b},+; c^{9}\right\rangle_{N S N S ; T}\right. \\
& \left.+\left|B(p+1), \mathbf{a}, \mathbf{b},-; c^{9}\right\rangle_{N S N S ; T}\right) \\
& \left|B(p+1), \mathbf{a}, \mathbf{b} ; c^{9}\right\rangle_{R R ; T}=\frac{1}{\sqrt{2}}\left(\left|B(p+1), \mathbf{a}, \mathbf{b},+; c^{9}\right\rangle_{R R ; T}\right. \\
& \left.+\left|B(p+1), \mathbf{a}, \mathbf{b},-; c^{9}\right\rangle_{R R ; T}\right),
\end{aligned}
$$

where

$$
\begin{aligned}
|B(p+1), \mathbf{a}, \mathbf{b}, \eta\rangle_{N S N S ; U}= & \mathcal{M} \int\left(\prod_{\mu \in \mathcal{D}_{c}} d k^{\mu} e^{i \mathbf{k} \cdot \mathbf{a}}\right)\left(\prod_{j=6}^{8} \sum_{m_{j} \in \mathbb{Z}} e^{i m_{j} b^{j} / R_{j}}\right) \\
& \sum_{n_{9} \in \mathbb{Z}}\left|B(p+1) \widehat{, \mathbf{k}}, \mathbf{m}, n_{9}, \eta\right\rangle_{N S N S ; U} \\
|B(p+1), \mathbf{a}, \mathbf{b}, \eta\rangle_{R R ; U}= & 4 i \mathcal{M} \int\left(\prod_{\mu \in \mathcal{D}_{c}} d k^{\mu} e^{i \mathbf{k} \cdot \mathbf{a}}\right)\left(\prod_{j=6}^{8} \sum_{m_{j} \in \mathbb{Z}} e^{i m_{j} b^{j} / R_{j}}\right) \\
& \sum_{n_{9} \in \mathbb{Z}}\left|B(p+1), \widehat{\mathbf{k}}, \mathbf{m}, n_{9}, \eta\right\rangle_{R R ; U} \\
\left|B(p+1), \mathbf{a}, \mathbf{b}, \eta ; c^{9}\right\rangle_{N S N S ; T}= & 2 \widetilde{\mathcal{M}} \int\left(\prod_{\mu \in \mathcal{D}_{c}} d k^{\mu} e^{i \mathbf{k} \cdot \mathbf{a}}\right)\left|B(p+1), \widehat{\mathbf{k}}, \mathbf{b}, \eta ; c^{9}\right\rangle_{N S N S ; T} \\
\left|B(p+1), \mathbf{a}, \mathbf{b}, \eta ; c^{9}\right\rangle_{R R ; T}= & 2 i \widetilde{\mathcal{M}} \int\left(\prod_{\mu \in \mathcal{D}_{c}} d k^{\mu} e^{i \mathbf{k} \cdot \mathbf{a}}\right)\left|B(p+1), \widehat{k}, \mathbf{b}, \eta ; c^{9}\right\rangle_{R R ; T} .
\end{aligned}
$$


$c^{9}$ in Eq. (3.16) takes values 0 or $\pi R_{9}$, and corresponds to twisted sector states associated to the fixed points $(\mathbf{b}, 0)$ and $\left(\mathbf{b}, \pi R_{9}\right)$, respectively. $n_{9}$ labels the winding number along $x^{9}$, and $\mathcal{D}_{c}$ is defined as before. The coherent momentum (and winding) eigenstates $\left|B(p+1) \widehat{, \mathbf{k}}, \mathbf{m}, n_{9}, \eta\right\rangle$ (in the case of the untwisted sectors) and $\left|B(p+1) \widehat{, \mathbf{k}}, \mathbf{b}, \eta ; c^{9}\right\rangle$ (in the case of the twisted sectors) are again given by the same formula as in (3.5), the only difference being that now $\mathcal{C}$ contains also $\mu=9$ and $\hat{\mathcal{D}}$ does not. Furthermore the moding of the fermions in the untwisted RR sector is opposite to that in the untwisted NSNS sector, and that in the twisted NSNS sector is opposite to that in the twisted RR sector discussed below (3.5).

The normalisation constants $\mathcal{M}$ and $\widetilde{\mathcal{M}}$ can be determined from the tree level diagram that describes the exchange of closed string states between two such branes. We take them to be located at the same point in the compact space (say at $\mathbf{b}=0$ ), but at different points $\mathbf{a}_{1}$ and $\mathbf{a}_{2}$ in the non-compact space. We also take them to carry the same twisted sector RR charges. The relevant term describing the interaction between a pair of branes of this type is

$$
\int_{0}^{\infty} d l\left\langle D(p+1), \mathbf{a}_{1}, \mathbf{b}_{1}=0, \epsilon_{1}, \kappa\left|e^{-l H_{c}}\right| D(p+1), \mathbf{a}_{2}, \mathbf{b}_{2}=0, \epsilon_{2}, \kappa\right\rangle
$$

which can be evaluated to give

$$
\begin{gathered}
\frac{1}{4} \int_{0}^{\infty} d l l^{-\frac{5-p}{2}} e^{-\frac{\left(\mathbf{a}_{1}-\mathbf{a}_{2}\right)^{2}}{4 \pi l}}\left[\mathcal{M}^{2}\left(\sum_{n_{9} \in \mathbb{Z}} e^{-l \pi R_{9}^{2} n_{9}^{2}}\right)\left(\prod_{i=6}^{8} \sum_{m_{i} \in \mathbb{Z}} e^{-l \pi m_{i}^{2} / R_{i}^{2}}\right)\right. \\
\times \frac{f_{3}^{8}(q)-f_{4}^{8}(q)-\epsilon_{1} \epsilon_{2} f_{2}^{8}(q)}{f_{1}^{8}(q)} \\
\left.-\widetilde{\mathcal{M}}^{2} \frac{f_{2}^{4}(q) f_{3}^{4}(q)}{f_{1}^{4}(q) f_{4}^{4}(q)}\left(1-\epsilon_{1} \epsilon_{2}\right)\right] .
\end{gathered}
$$

$\epsilon_{j}= \pm$ distinguishes between brane or anti-brane for the branes localised at $\mathbf{a}_{j}, j=1,2$. Again $q=e^{-2 \pi l}$, and the first two lines come from the untwisted NSNS and RR sector, whereas the last line combines the contributions from the two twisted NSNS and RR sectors at $x^{9}=0$ and $x^{9}=\pi R_{9}$. Using (2.13) it is clear that the amplitude vanishes identically if the system preserves supersymmetry, i.e. for $\epsilon_{1}=\epsilon_{2}$.

We can rewrite the amplitude again in terms of open string coordinates by setting 
$t=1 / 2 l$. Then (3.18) becomes

$$
\begin{aligned}
& \frac{1}{4} \int_{0}^{\infty} \frac{d t}{2 t} \tilde{q}^{\frac{\left(\mathbf{a}_{1}-\mathbf{a}_{2}\right)^{2}}{2 \pi^{2}}} t^{-\frac{p+1}{2}} {\left[\mathcal{M}^{2} 2^{(9-p) / 2} \frac{R_{6} R_{7} R_{8}}{R_{9}}\left(\sum_{m_{9} \in \mathbb{Z}} \tilde{q}^{2 m_{9}^{2} / R_{9}^{2}}\right)\right.} \\
& \times\left(\prod_{i=6}^{8} \sum_{n_{i} \in \mathbb{Z}} \tilde{q}^{2 n_{i}^{2} R_{i}^{2}}\right) \frac{f_{3}^{8}(\tilde{q})-f_{2}^{8}(\tilde{q})-\epsilon_{1} \epsilon_{2} f_{4}^{8}(\tilde{q})}{f_{1}^{8}(\tilde{q})} \\
&\left.-\widetilde{\mathcal{M}}^{2} 2^{(5-p) / 2} \frac{f_{4}^{4}(\tilde{q}) f_{3}^{4}(\tilde{q})}{f_{1}^{4}(\tilde{q}) f_{2}^{4}(\tilde{q})}\left(1-\epsilon_{1} \epsilon_{2}\right)\right]
\end{aligned}
$$

where we have again used (3.9) and (3.10). For $\epsilon_{1}=-\epsilon_{2}$ this agrees with (2.27) provided that

$$
32 \frac{R_{6} R_{7} R_{8}}{R_{9}} \mathcal{M}^{2}=\frac{A}{(2 \pi)^{p+1}}, \quad 2 \widetilde{\mathcal{M}}^{2}=\frac{A}{(2 \pi)^{p+1}} .
$$

Now that we have determined the constants, (3.18) becomes

$$
\begin{gathered}
\frac{1}{8} \frac{A}{(2 \pi)^{p+1}} \int_{0}^{\infty} d l l^{-\frac{5-p}{2}} e^{-\frac{\left(\mathbf{a}_{1}-\mathbf{a}_{2}\right)^{2}}{4 \pi l}}\left[\frac{1}{16} \frac{R_{9}}{R_{6} R_{7} R_{8}}\left(\sum_{n_{9} \in \mathbb{Z}} e^{-l \pi R_{9}^{2} n_{9}^{2}}\right)\left(\prod_{i=6}^{8} \sum_{m_{i} \in \mathbb{Z}} e^{-l \pi m_{i}^{2} / R_{i}^{2}}\right)\right. \\
\left.\frac{f_{3}^{8}(q)-f_{4}^{8}(q)-\epsilon_{1} \epsilon_{2} f_{2}^{8}(q)}{f_{1}^{8}(q)}-\frac{f_{2}^{4}(q) f_{3}^{4}(q)}{f_{1}^{4}(q) f_{4}^{4}(q)}\left(1-\epsilon_{1} \epsilon_{2}\right)\right] .
\end{gathered}
$$

For $\epsilon_{1}=-\epsilon_{2}$, using (2.13), the integrand simplifies to

$$
\begin{gathered}
\frac{1}{4} \frac{A}{(2 \pi)^{p+1}} l^{-\frac{5-p}{2}} e^{-\frac{\left(\mathbf{a}_{1}-\mathbf{a}_{2}\right)^{2}}{4 \pi l}}\left[\frac{R_{9}}{16 R_{6} R_{7} R_{8}}\left(\sum_{n_{9} \in \mathbb{Z}} e^{-l \pi R_{9}^{2} n_{9}^{2}}\right)\left(\prod_{i=6}^{8} \sum_{m_{i} \in \mathbb{Z}} e^{-l \pi m_{i}^{2} / R_{i}^{2}}\right) \frac{f_{2}^{8}(q)}{f_{1}^{8}(q)}\right. \\
\left.-\frac{f_{2}^{4}(q) f_{3}^{4}(q)}{f_{1}^{4}(q) f_{4}^{4}(q)}\right] .
\end{gathered}
$$

At the critical radius, $R_{6}=R_{7}=R_{8}=1 / \sqrt{2}, R_{9}=\sqrt{2}$, the momentum and winding sums simplify as before using (2.18), and (3.22) vanishes.

From the point of view of the closed string calculation, the first term in the integrand of (3.21) contains the gravitational interaction and the interaction due to the exchange of the untwisted RR fields. In the case of the brane-anti-brane pair, the two branes have opposite charge with respect to the untwisted RR fields, and therefore both interactions are attractive. Indeed, for $\epsilon_{1}=-\epsilon_{2}$ the last factor in the first term in (3.21) is $2 f_{2}^{8} / f_{1}^{8}$, and so the first term is strictly positive. The second term contains the interaction due to the states in the twisted sectors. For the case of brane-anti-brane pair (without Wilson line) the interactions at both fixed points of $T^{4} / g$ are equal and repulsive. This is due to 
the fact that the two states under consideration carry the opposite twisted NSNS and the same twisted RR charge at each fixed point; both lead to a repulsive force. At the critical point the attractive interaction due to the untwisted sector fields cancels the repulsive interaction due to the twisted sector fields at all distance scales.

\subsection{Example 3: A pair of BPS D-branes wrapped on non-super- symmetric cycles of K3}

The theory under consideration is again type IIB/IIA on $T^{4}$ modded out by $\mathcal{I}_{4} \cdot(-1)^{F_{L}}$. We shall take a pair of non-BPS D $p$-branes of the type described in section 3.1, one at $(\mathbf{a}, \mathbf{b}=0)$ and the other at $\left(\mathbf{a}, \mathbf{b}=\left(0,0,0, \pi R_{9}\right)\right)$. Note that we have taken the locations of the two branes in the non-compact directions to be identical. In the dual type IIA/IIB string theory on $T^{4} / \mathcal{I}_{4}$, this describes a system containing a pair of non-BPS branes, corresponding to BPS D-branes wrapped on two homologically distinct non-supersymmetric 2-cycles, situated at the same location in the non-compact space-time. The boundary state describing this system is given by:

$$
\left|\widetilde{D} p_{1}, \widetilde{D} p_{2}\right\rangle=\left|\widetilde{D} p, \mathbf{a}, \mathbf{b}=0, \epsilon_{1}\right\rangle+\left|\widetilde{D} p, \mathbf{a}, \mathbf{b}=\left(0,0,0, \pi R_{9}\right), \epsilon_{2}\right\rangle
$$

where the boundary states appearing on the right hand side of this equation are identical to the ones defined in section 3.1. The amplitude of interest is

$$
\int_{0}^{\infty} d l\left\langle\widetilde{D} p_{1}, \widetilde{D} p_{2}\left|e^{-l H_{c}}\right| \widetilde{D} p_{1}, \widetilde{D} p_{2}\right\rangle
$$

This can be computed easily using the expression for the boundary states given in section 3.1, Eqs. (3.1)-3.5), and is given by

$$
\begin{aligned}
& \frac{1}{2} \int_{0}^{\infty} d l \quad l^{-\frac{5-p}{2}} \quad\left[4 \mathcal{N}^{2}\left(\prod_{i=6}^{8} \sum_{m_{i} \in \mathbb{Z}} e^{-l \pi m_{i}^{2} / R_{i}^{2}}\right)\left(\sum_{m_{9} \in 2 \mathbb{Z}} e^{-l \pi m_{9}^{2} / R_{9}^{2}}\right) \frac{f_{3}^{8}(q)-f_{4}^{8}(q)}{f_{1}^{8}(q)}\right. \\
& \left.-2 \widetilde{\mathcal{N}}^{2} \frac{f_{2}^{4}(q) f_{3}^{4}(q)}{f_{1}^{4}(q) f_{4}^{4}(q)}\right],
\end{aligned}
$$

where $\mathcal{N}$ and $\widetilde{\mathcal{N}}$ have been defined in (3.12). Using these, (3.25) can be rewritten as

$$
\begin{aligned}
& \frac{1}{2} \frac{A}{(2 \pi)^{p+1}} \int_{0}^{\infty} d l l^{-\frac{5-p}{2}} \\
& {\left[\frac{1}{8 R_{6} R_{7} R_{8} R_{9}}\left(\prod_{i=6}^{8} \sum_{m_{i} \in \mathbb{Z}} e^{-l \pi m_{i}^{2} / R_{i}^{2}}\right)\left(\sum_{m_{9} \in 2 \mathbb{Z}} e^{-l \pi m_{9}^{2} / R_{9}^{2}}\right) \frac{f_{3}^{8}(q)-f_{4}^{8}(q)}{f_{1}^{8}(q)}-\frac{f_{2}^{4}(q) f_{3}^{4}(q)}{f_{1}^{4}(q) f_{4}^{4}(q)}\right] .}
\end{aligned}
$$


By the same identities used in section 3.1 we see that this vanishes exactly at

$$
R_{6}=R_{7}=R_{8}=\frac{1}{\sqrt{2}}, \quad R_{9}=\sqrt{2} .
$$

Thus at this critical point the spectrum of open string states on this system develops exact Bose-Fermi degeneracy.

Note that at this critical radius there is an exact marginal deformation which takes this system to the system discussed in section 3.2 [15, 17]. Thus it is natural to ask if the bose-fermi degeneracy of the spectrum survives all along this line of marginal deformation. This is however not the case. From the arguments in Ref. [17] it is easy to see that the spectrum in the Ramond sector does not change during this marginal deformation, whereas the spectrum in the NS sector certainly does. Thus the bose-fermi degeneracy in the spectrum can only appear at special points along this critical line.

\subsection{Example 4: A pair of BPS D-branes wrapped on supersym- metric cycles of $\mathrm{K} 3$}

This example will involve a system similar to that discussed in section 3.2, - the only difference being that instead of taking a pair of anti-parallel BPS $\mathrm{D}(p+1)$ branes in type IIB/IIA on $T^{4}$ modded out by $(-1)^{F_{L}} \cdot \mathcal{I}_{4}$, we shall consider a pair of 'orthogonal' BPS $\mathrm{D}(p+1)$ branes. 9 In particular we shall take both $\mathrm{D}$-branes to span the same non-compact directions, but take the first D-brane to lie along $x^{9}$ at $\mathbf{b} \equiv\left(x^{6}=0, x^{7}=0, x^{8}=0\right)$, and the second D-brane to lie along $x^{8}$ at $\mathbf{b}^{\prime} \equiv\left(x^{6}=0, x^{7}=0, x^{9}=0\right)$. If $\kappa$ denotes the sign of the twisted RR charge of the first D-brane at the fixed points $(0,0,0,0)$ and $\left(0,0,0, \pi R_{9}\right)$, and $\kappa^{\prime}$ denotes the sign of the twisted RR charge of the second D-brane at the fixed points $(0,0,0,0)$ and $\left(0,0, \pi R_{8}, 0\right), \mathbf{a}$ and $\mathbf{a}^{\prime}$ denote their locations in the non-compact directions, and $\epsilon, \epsilon^{\prime}$ denote the sign of the untwisted sector RR charges, then the boundary state of the combined system is given by

$$
|D(p+1), \mathbf{a}, \mathbf{b}, \epsilon, \kappa\rangle+\left|D(p+1), \mathbf{a}^{\prime}, \mathbf{b}^{\prime}, \epsilon^{\prime}, \kappa^{\prime}\right\rangle^{\prime},
$$

where $|D(p+1), \mathbf{a}, \mathbf{b}, \epsilon, \kappa\rangle$ is the boundary state defined in section 3.2, and $\mid D(p+$ 1), $\left.\mathbf{a}^{\prime}, \mathbf{b}^{\prime}, \epsilon^{\prime}, \kappa^{\prime}\right\rangle^{\prime}$ is related to the boundary state defined in section 3.2 by exchanging the 8th and the 9th coordinates everywhere.

\footnotetext{
${ }^{6}$ Some aspects of tachyon condensation on D-branes at angles have been recently discussed in [27.
} 
In computing the amplitude describing the emission and reabsorption of closed strings from this system, we note that since each system is individually BPS the amplitude for emission and reabsorption of a closed string by the same D-brane will vanish identically. Thus the relevant amplitude which needs to be analysed is

$$
\int_{0}^{\infty} d l\left\langle D(p+1), \mathbf{a}, \mathbf{b}, \epsilon, \kappa\left|e^{-l H_{c}}\right| D(p+1), \mathbf{a}^{\prime}, \mathbf{b}^{\prime}, \epsilon^{\prime}, \kappa^{\prime}\right\rangle^{\prime} .
$$

Each of the boundary states in this expression has components in the untwisted NSNS and RR sectors, as well as the twisted NSNS and RR sectors. The calculation can be simplified by noting that in any sector which contains zero modes of the fermion fields $\psi^{8}, \psi^{9}, \widetilde{\psi}^{8}, \widetilde{\psi}^{9}$, the two Fock vacua appearing in the expression for the two boundary states in Eq. (3.29) are orthogonal since they satisfy different constraints coming from the fermionic zero modes in the analogue of Eq. (3.2). Thus the contribution to (3.29) from these sectors vanishes. This leaves us with the untwisted NSNS and the twisted RR sectors. Furthermore, among the various twisted sector RR states appearing in (3.28), only the sector that is localised at $x^{6}=x^{7}=x^{8}=x^{9}=0$ contributes to (3.29), since this is the only sector that is shared by both boundary states. The contribution can be easily evaluated, and gives

$$
\begin{aligned}
& \frac{1}{4} \int d l e^{-\frac{\left(\mathbf{a}-\mathbf{a}^{\prime}\right)^{2}}{4 \pi l}} l^{-\frac{5-p}{2}}\left[2 \mathcal{M} \mathcal{M}^{\prime}\left(\sum_{m_{6}, m_{7} \in \mathbb{Z}} e^{-l \pi\left(\left(m_{6} / R_{6}\right)^{2}+\left(m_{7} / R_{7}\right)^{2}\right)}\right)\right. \\
& \left.\times \frac{f_{3}(q)^{6} f_{4}(q)^{2}-f_{4}(q)^{6} f_{3}(q)^{2}}{f_{1}(q)^{6} f_{2}(q)^{2}}-\frac{1}{2} \kappa \kappa^{\prime} \widetilde{\mathcal{M}} \widetilde{\mathcal{M}}^{\prime} \frac{f_{2}(q)^{4} f_{3}(q)^{2} f_{4}(q)^{2}}{f_{1}(q)^{4} f_{4}(q)^{2} f_{3}(q)^{2}}\right],
\end{aligned}
$$

where $\mathcal{M}, \widetilde{\mathcal{M}}$ are given as in (3.20), and $\mathcal{M}^{\prime}, \widetilde{\mathcal{M}}^{\prime}$ are obtained from Eq. (3.20) by exchanging $8 \leftrightarrow 9$ :

$$
\mathcal{M}^{2}=\frac{A}{(2 \pi)^{p+1}} \frac{R_{9}}{32 R_{6} R_{7} R_{8}} \quad \mathcal{M}^{\prime 2}=\frac{A}{(2 \pi)^{p+1}} \frac{R_{8}}{32 R_{6} R_{7} R_{9}},
$$

and

$$
\widetilde{\mathcal{M}}^{2}=\widetilde{\mathcal{M}}^{\prime}=\frac{A}{2(2 \pi)^{p+1}} .
$$

The integral (3.30) then becomes

$$
\begin{aligned}
I= & \frac{1}{16} \frac{A}{(2 \pi)^{p+1}} \int_{0}^{\infty} d l e^{-\frac{\left(\mathbf{a}-\mathbf{a}^{\prime}\right)^{2}}{4 \pi l}} l^{-\frac{5-p}{2}} \\
& {\left[\frac{1}{4 R_{6} R_{7}}\left(\sum_{m_{6}, m_{7}} e^{-l \pi\left(\left(m_{6} / R_{6}\right)^{2}+\left(m_{7} / R_{7}\right)^{2}\right)}\right) \frac{f_{3}(q)^{6} f_{4}(q)^{2}-f_{4}(q)^{6} f_{3}(q)^{2}}{f_{1}(q)^{6} f_{2}(q)^{2}}-\kappa \kappa^{\prime} \frac{f_{2}(q)^{4}}{f_{1}(q)^{4}}\right] . }
\end{aligned}
$$


At the 'critical radius' $R_{6}=R_{7}=1 / 2$, the sums over $m_{6}, m_{7}$ give

$$
\sum_{m_{6}, m_{7}} e^{-l \pi\left(\left(m_{6} / R_{6}\right)^{2}+\left(m_{7} / R_{7}\right)^{2}\right)}=\left(\sum_{n} q^{2 n^{2}}\right)^{2} .
$$

This can be re-expressed in terms of the Jacobi $\vartheta$-function $\vartheta_{3}(0 \mid \tau)$ that we considered before in Eq. (2.16), and the Jacobi $\vartheta$-function $\vartheta_{4}(0 \mid \tau)$, whose sum and product representation is 21 .

$$
\vartheta_{4}(0 \mid \tau)=\sum_{n \in \mathbb{Z}} q^{n^{2}}(-1)^{n}=\prod_{n=1}^{\infty}\left(1-q^{2 n}\right)\left(1+q^{2 n-1}\right)^{2}=f_{1}(q) f_{4}^{2}(q) .
$$

Indeed, using (2.16) and (3.35), we find

$$
\begin{aligned}
\vartheta_{3}^{2}(0 \mid \tau)+\vartheta_{4}^{2}(0 \mid \tau) & =\sum_{n, l} q^{n^{2}+l^{2}}+\sum_{n, l}(-1)^{n+l} q^{n^{2}+l^{2}} \\
& =\sum_{n, l}\left(1+(-1)^{n+l}\right) q^{\frac{1}{2}\left[(n+l)^{2}+(n-l)^{2}\right]} \\
& =2 \sum_{r, \text { seven }} q^{\frac{1}{2}\left[r^{2}+s^{2}\right]} \\
& =2\left(\sum_{m} q^{2 m^{2}}\right)^{2},
\end{aligned}
$$

where we have set $r=n+l$ and $s=n-l$, and observed that if $r=n+l$ is even, then so is $s=n-l$. Thus (3.34) can be rewritten as

$$
\left(\sum_{n} q^{2 n^{2}}\right)^{2}=\frac{1}{2} f_{1}(q)^{2}\left(f_{3}(q)^{4}+f_{4}(q)^{4}\right),
$$

and the integrand in (3.33) becomes, apart from the overall factor of $\frac{1}{16} \frac{A}{(2 \pi)^{p+1}} e^{\frac{-\left(\mathbf{a}-\mathbf{a}^{\prime}\right)^{2}}{4 \pi l}} l^{-\frac{5-p}{2}}$ and for $\kappa \kappa^{\prime}=1$,

$$
\begin{aligned}
& {\left[\frac{1}{2} \frac{f_{3}(q)^{2} f_{4}(q)^{2}\left(f_{3}(q)^{4}-f_{4}(q)^{4}\right)\left(f_{3}(q)^{4}+f_{4}(q)^{4}\right)}{f_{1}(q)^{4} f_{2}(q)^{2}}-\frac{f_{2}(q)^{4}}{f_{1}(q)^{4}}\right]} \\
& =\frac{1}{f_{1}(q)^{4} f_{2}(q)^{2}}\left[\frac{1}{2} f_{3}(q)^{2} f_{4}(q)^{2}\left(f_{3}(q)^{8}-f_{4}(q)^{8}\right)-f_{2}(q)^{6}\right] \\
& =\frac{f_{2}(q)^{6}}{f_{1}(q)^{4} f_{2}(q)^{2}}\left[\frac{1}{2}\left(f_{3}(q)^{2} f_{4}(q)^{2} f_{2}(q)^{2}\right)-1\right]=0,
\end{aligned}
$$

where we have used the identity (2.17) in the last line. 
The radius $R_{6}=R_{7}=1 / 2$ is critical in the sense that for $R_{i}<1 / 2, i=6,7$, the open string that begins on one $\mathrm{D}(p+1)$-brane and ends on the other contains a tachyon. Indeed, in this case the $C_{o}$ in Eq. (2.2) is equal to -(1/4), and the energy of the winding states with winding number $\left(n_{6}, n_{7}\right)$ along the 6 th and the 7 th directions is given by

$$
-\frac{\pi}{4}+\pi\left(n_{6} R_{6}\right)^{2}+\pi\left(n_{7} R_{7}\right)^{2} .
$$

The $n_{6}=n_{7}=0$ mode is projected out, but appropriate linear combinations of the states with $\left(n_{6}, n_{7}\right)=( \pm 1,0)$ or $\left(n_{6}, n_{7}\right)=(0, \pm 1)$ survive. At least one of them is tachyonic whenever $R_{6}$ or $R_{7}$ is below (1/2), and the system is therefore unstable in this regime. For generic values of the radii $R_{8}$ and $R_{9}$ there does not seem to be any simple system of D-branes into which this system can decay. This leads us to suspect that below the critical values of $R_{6}$ and/or $R_{7}$ the system forms a bound state which cannot be described by a solvable boundary conformal field theory of a system of D-branes.

\section{Acknowledgement}

This work was begun during the Extended Workshop on String Theory that was held at The Abdus Salam International Centre for Theoretical Physics, Trieste in June-July 1999. We would like to thank the ICTP for hospitality during this workshop.

M.R.G. is supported by a College Lectureship of Fitzwilliam College, Cambridge. 


\section{References}

[1] A. Sen, JHEP 12, 021 (1998); hep-th/9812031.

[2] O. Bergman and M.R. Gaberdiel, JHEP 03, 013 (1999); hep-th/9901014.

[3] M.R. Douglas and G. Moore, hep-th/9603167.

[4] I. Antoniadis, E. Dudas and A. Sagnotti, hep-th/9908023.

[5] S. Kachru, J. Kumar and E. Silverstein, Phys. Rev. D59, 106004 (1999); hepth/9807076.

[6] J.A. Harvey, Phys. Rev. D59, 026002 (1999); hep-th/9807213.

[7] S. Kachru and E. Silverstein, JHEP 11, 001 (1998); hep-th/9808056.

[8] G. Shiu and S.H. Tye, Nucl. Phys. B542, 45 (1999); hep-th/9808095.

[9] S. Kachru and E. Silverstein, JHEP 01, 004 (1999); hep-th/9810129.

[10] C. Angelantonj, I. Antoniadis and K. Forger, hep-th/9904092.

[11] J. Maldacena, Adv. Theor. Math. Phys. 2, 231 (1998); hep-th/9711200.

[12] S.S. Gubser, I.R. Klebanov and A.M. Polyakov, Phys. Lett. B428, 105 (1998); hepth/9802109.

[13] E. Witten, Adv. Theor. Math. Phys. 2, 253 (1998); hep-th/9802150.

[14] M.B. Green and M. Gutperle, Nucl. Phys. B476, 484 (1996); hep-th/9604091.

[15] A. Sen, JHEP 08, 010 (1998); hep-th/9805019.

[16] O. Bergman and M.R. Gaberdiel, Phys. Lett. B441, 133 (1998); hep-th/9806155.

[17] A. Sen, JHEP 09, 023 (1998); hep-th/9808141.

[18] A. Sen, JHEP 10, 021 (1998); hep-th/9809111.

[19] A. Sen, hep-th/9904207. 
[20] J. Polchinski and Y. Cai, Nucl. Phys. B296, 91 (1988).

[21] A. Erdéli, W. Magnus, F. Oberhettinger and F. G. Tricomi, Higher Transcendental Functions, Vol. 2, McGraw-Hill, (1953); p. 354 ff.

[22] C. Callan, C. Lovelace, C. Nappi and S. Yost, Nucl. Phys. B308, 221 (1988).

[23] T. Onogi and N. Ishibashi, Mod. Phys. Lett. A4, 161 (1989).

[24] N. Ishibashi, Mod. Phys. Lett. A4, 251 (1989).

[25] C. Lovelace, Phys. Lett. B34, 500 (1971).

L. Clavelli and J. Shapiro, Nucl. Phys. B57, 490 (1973).

M. Ademollo, R. D'Auria, F. Gliozzi, E. Napolitano, S. Sciuto and P. di Vecchia, Nucl. Phys. B94, 221 (1975).

C. Callan, C. Lovelace, C. Nappi and S. Yost, Nucl. Phys. B293, 83 (1987).

M. Bianchi and A. Sagnotti, Phys. Lett. 247B, 517 (1990); Nucl. Phys. B361, 519 (1991).

P. Horava, Nucl. Phys. B327, 461 (1989).

[26] O. Bergman and M.R. Gaberdiel, Nucl. Phys. B499, 183 (1997); hep-th/9701137.

M. Li, Nucl. Phys. B460, 351 (1996); hep-th/9510161.

H. Ooguri, Y. Oz and Z. Yin, Nucl. Phys. B477, 407 (1996); hep-th/9606112.

K. Becker, M. Becker, D. Morrison, H. Ooguri, Y. Oz and Z. Yin, Nucl. Phys. B480, 225 (1996); hep-th/9608116.

M. Kato and T. Okada, Nucl. Phys. B499, 583 (1997); hep-th/9612148.

S. Stanciu, Nucl. Phys. B526, 295 (1998); hep-th/9708166.

A. Recknagel and V. Schomerus, Nucl. Phys. B531, 185 (1998); hep-th/9712186.

J. Fuchs and C. Schweigert, Nucl. Phys. B530, 99 (1998); hep-th/9712257.

S. Stanciu and A. Tseytlin, JHEP 06, 010 (1998); hep-th/9805006.

M. Gutperle and Y. Satoh, Nucl. Phys. B543, 73 (1999); hep-th/9808080.

F. Hussain, R. Iengo, C. Nunez and C. Scrucca, Phys. Lett. B409, 101 (1997); hepth/9706186.

M. Bertolini, R. Iengo and C. Scrucca, Nucl. Phys. B522, 193 (1998); hepth/9801110. 
M. Bertolini, P. Fre, R. Iengo and C. Scrucca, Phys. Lett. B431, 22 (1998); hepth/9803096.

P. Di Vecchia, M. Frau, A. Lerda, I. Pesando, R. Russo and S. Sciuto, Nucl. Phys. B507, 259 (1997); hep-th/9707068.

M. Billo, P. Di Vecchia, M. Frau, A. Lerda, I. Pesando, R. Russo and S. Sciuto, Nucl. Phys. B526, 199 (1998); hep-th/9802088.

A. Recknagel and V. Schomerus, Nucl. Phys. B545, 233 (1999); hep-th/9811237.

[27] S.P. de Alwis, hep-th/9905080. 